\title{
Biomarkers and their Role in Premonition, Interpretation and Treatment of Cancer
}

\author{
Sai YRKM ${ }^{1 *}$, Dattatreya $A^{1}$, Anand $S Y^{1}$ and Mahalakshmi $D^{2}$
}

${ }^{1}$ GITAM Institute of Sciences, GITAM University, Vishakhapatnam, A.P, India

${ }^{2}$ KGRL Degree \& PG College, Affiliated to Andhra University, Bhimavaram, India

\begin{abstract}
The Prognosis and Diagnosis of CANCER has become easier now days, in earlier days, there has been a degree of difficulty in the premonition and Interpretation of the disease, either of the carcinomas, sarcomas, lymphomas and/ or etc cancers types are very dreadful diseases which do not have methods of earlier detections. Biomarkers' are the astounding promise for both of the Diagnosis and Prognosis of the cancer. They set a future for the further eagle eye study of various cancers. In this review it will be stating various types of biomarkers whether they are immunological origin or of molecular origin. In the finale statement is we tried to summarize and hypothesize the types in the biomarkers' for the prognosis and diagnosis of Cancer.
\end{abstract}

Keywords: Prostate cancer; Biomarkers'; Prognostic studies; Novel diagnostic methods

\section{Introduction}

Cancer is the state of cells marked by unregulated proliferation. There are several characteristic features attributed to its occurrence so as to demarcate itself from the normal tissue system, and one of them is Angiogenesis, that ensures its continued survival in the body. Angiogenesis, in the recent past has become a nucleus of notice and has hence opened new portals in the realm of cancer therapy. AntiAngiogenic studies have made hysteric escalations in the evolution of effectual anti-cancer drugs [10].

A biomarker can be a substance that is introduced into an organism as a means to examine organ function or other aspects of health. For example, rubidium chloride is used as a radioactive isotope to evaluate perfusion of heart muscle. It can also be a substance whose detection indicates a particular disease state, for example, the presence of an antibody may indicate an infection. More specifically, a biomarker indicates a change in expression or state of a protein that correlates with the risk or progression of a disease, or with the susceptibility of the disease to a given treatment. Biomarkers are characteristic biological properties that can be detected and measured in parts of the body like the blood or tissue. They may indicate either normal or diseased processes in the body [2]. Biomarkers can be specific cells, molecules, or genes, gene products, enzymes, or hormones. Complex organ functions or general characteristic changes in biological structures can also serve as biomarkers. Although the term biomarker is relatively new, biomarkers have been used in pre-clinical research and clinical diagnosis for a considerable time [3]. For example, body temperature is a well-known biomarker for fever. Blood pressure is used to determine the risk of stroke. It is also widely known that cholesterol values are a biomarker and risk indicator for coronary and vascular disease, and that C-reactive protein (CRP) is a marker for inflammation. As in this study we study about the Cancer Biomarkers. We should know about the gist on these biomarkers'

\section{Biomarkers}

The study of cancer biomarker proteins began in 1847 with the discovery by Henry Bence-Jones of what turned out, more than 100 years later, to be a tumor-produced free antibody light chain "Bence -Jones protein" in the urine of a multiple myeloma patient $[101,102]$ where it was present in large quantities and could be revealed by simple heat denaturation: One hundred and 40 years later this protein was demonstrated to be present also in the serum [4], and in 1998 a routine immunodiagnostic test was approved by the FDA: Hormones produced by tumors were also detected early on [100]. AdrenoCorticoTropic Hormone (ACTH), Calcitonin, and Chorionic Gonadotropin (hCG), for example, are elevated in specific cancer types, though not with the tumor specificity of Bence-Jones proteins [1].

Some of the bio-indicators or most probably called as biomarkers' of Cancer will be discussed in this review along with that some of the factors like, their role in Prognosis or premonition and also their role in diagnosis of cancer, and treatment to cancer basing on the biomarkers and other factors were discussed.

Biomarkers of Different origins within the body: The cancer stem cell theory proposes that there is a small but constant subpopulation of cancer cells with stem cell properties responsible for the self renewal capacity and unlimited proliferation of tumor as well as increased resistance to Antineoplastic drugs. Targeting these cells might constitute an effective way to cure cancer [2]. This make the Glioma cells as ingenuous cancer cells of the body and their population count can be calculated as the spectacular biomarker's for the prognosis of overall cancer cells.

Bile Acids: Promoters or Carcinogens in Colon Cancer? Since 1970s it has been generally considered that bile acids are promoters of colon cancer, but lack carcinogenic activity. However, recent evidence indicates that bile acids do, indeed, act as carcinogens. Substantial evidence from a variety of sources indicates that bile acids are involved in the etiology of colon cancer, although it has usually been assumed

${ }^{*}$ Corresponding author: Sai YRKM, GITAM Institute of Sciences, GITAM University, Vishakapatnam, A.P, India, E-mail: saiyrkm2454@gmail.com

Received June 15, 2011; Accepted July 20, 2011; Published July 22, 2011

Citation: Sai YRKM, Dattatreya A, Anand SY, Mahalakshmi D (2011) Biomarkers and their Role in Premonition, Interpretation and Treatment of Cancer. J Cancer Sci Ther S17. doi:10.4172/1948-5956.S17-002

Copyright: (c) 2011 Sai YRKM, et al. This is an open-access article distributed under the terms of the Creative Commons Attribution License, which permits unrestricted use, distribution, and reproduction in any medium, provided the original author and source are credited. 
that they only act as promoters. The incidence of colon cancer, as well as mortality rate, varies dramatically across regions of the world [6].

Prostate cancer (PC) is the second leading cause of cancer deaths in men. Epidemiological studies suggest that prostate cancer incidence \& increased in last few years in Asia. The causes or consequences of increasing trend of prostate cancer incidence are not completely known. Emerging evidences suggest that among the many risk factors, inflammation is the major risk factor for developing prostate cancer and its progression to metastasis; secondly Mature Bone-Producing Osteoblasts alter Gene Expression of Metastasis Related Genes in them. Prostate cancers often metastasize to bone. It is hypothesized that interactions between osteoblasts and cancer cells lead to establishment of prostate cancer skeletal metastasis. Interactions between prostate cancer cells and mature bone-producing osteoblasts resulted in gene expressional changes that may increase cells' ability to establish bone metastasis. The potential for inducing expressional changes seems to be crucial dependent of the differentiation status of osteoblasts [12]. It is proposed that exposure to environmental factors such as infectious agents, dietary agents and saturated lipids leads to injury of the prostate due to chronic inflammation and regenerative risk factor lesions referred to as proliferative inflammatory atrophy (PIA). These phenomena predominantly control by a number of pro-inflammatory macromolecules such as chemokines, and their receptors. Some recent studies suggest that many of these pro-inflammatory chemokines and their receptors are the products of proto-oncogenes in many cancers including that of the prostate. An understanding of this axis may enable researchers to develop targeted strategies and their Assay for their estimation can be a bio- indicator experiment for prostate cancer [4]. There's a study of phenomenon where in, a nanoparticle had been developed based imaging agent that targets SPARC (Secreted Protein Acidic Rich in Cysteine), a molecular marker of prostate cancer metastatic potential. The SPARC-targeted peptide sequence was used to design a biomaterial with improved pharmacokinetic properties by attaching it to a biocompatible nanoparticle that is also coupled to a fluorophore for in vivo imaging. Prostate cancer cell lines with varying degrees of SPARC expression were used to show the ability of the targeted nanoparticle to bind specifically to SPARC in vitro and in vivo including the clinically relevant bone and lung metastases. We show that in vivo imaging information correlates with the metastatic potential of the prostate tumor. This prognostic information could enable doctors to stratify patients and design personalized treatment strategies [8]. Another detection method is based on the Quadriplex model which is urine based method, The major advantages of urine-based assays are their non-invasive character and ability to monitor prostate cancer (CaP) with heterogeneous foci [60].

The introduction of mass screening programmes has been controversial. PSA (prostate-specific antigen) detection technology. The controversy is moving into a new phase, associated with the development of new molecular genetics biomarkers and tests derived from genome-association studies [53]. In this screening programs influence of chronic prostatitis on serum PSA level is well studied. What has to be determined is, whether it also influences potential new biomarkers of prostate cancer $(\mathrm{PCa}$ ) or not. In this prospective study to evaluate the effect of chronic prostatitis on the PCa urinary marker PCA3 NIH II and III chronic prostatitis did not influence the PCA3 score [70]. PSA glycosylation seems to depend on the pathophysiological conditions of the individual. Thus, methods to separate PSA isoforms (peaks) to study their role as PCa markers are needed. CE methods for analysis of isoforms of prostate-specific antigen have been developed as one of the detection methods. Prostate-specific antigen (PSA) is the usual biomarker for prostate cancer (PCa). However, its lack of selectivity has lead to the search for new biomarkers. CE methods for PSA isoforms separation, based on the use of different dynamic coatings, are developed using UV detection [77].

Besides these studies, Phosphoproteome analysis and DNA methylome [79] of the prostate cancer tissue specimens were studied, the former is by A gel-free proteomic strategy included whole proteome digestion, phosphopeptide enrichment with immobilized metal ion affinity chromatography (IMAC), and phosphoprotein identification via LC-MS/MS and database searches. The study showed that cancerrelevant phosphoproteins can be characterized directly from archived prostate tumor specimens, establishing the groundwork for further biomarker discovery [72].

Biomarkers' in Mouth, Larynx and Nasopharynx carcinomas: Prediction of which laryngeal dysplasia cases will progress to cancer is poorly achieved. The differential expression of biomarkers in dysplastic and cancerous lesions may help to improve this. Currently there is no good evidence for the use of biomarkers in predicting the future behavior of laryngeal dysplastic lesions [71].

Hair pin short RNA's [81] and the micro RNA's [80] (Profiling of Epstein-Barr virus-encoded) were respectively acted as the biomarkers for the Oral squamous cancer and Nasopharyngeal carcinoma and also in the human colorectal cancer [91]. MicroRNA (miRNA), non-coding RNA of approximately 22 nucleotides, post-transcriptionally represses expression of its target genes, miRNA regulates a variety of biological processes such as cell proliferation, cell death, development, stemness and genomic stability, not only in physiological conditions but also in various pathological conditions such as cancers [92]. Identification of novel cancer-associated miRNA seems promising considering their possible application in the development of novel cancer therapies and biomarkers. Currently, there are two major approaches to identify miRNA that are associated with cancer: expression profiling study and functional screening assay. The combination of both types of approaches will contribute to further elucidation of the cancer pathophysiology and to the development of a novel class of cancer therapies and biomarkers [92].

Serological antibodies were also potential biomarkers' for Oropharyngeal Cancer, where the HPV16 proteome is associated with OPC. Here Patients with HPV16+ OPC have detectable Abs to E1, E2, and E7 proteins, which are potential biomarkers for HPV-associated OPC [95].

Cancer Biomarkers' from that of Immunological origin: CD133 has been used to identify normal and cancer stem cells from several different tissues. Nowadays some researchers have reported that CD133 expression was not restricted to cancer stem cells (CSCs) of colorectal cancer and brain tumors, and CD133-negative subsets could also initiate tumors. Therefore a meta-analysis to assess the value of CD133 as a biomarker of CSCs for colorectal cancer and brain tumors has been performed [61]

Standard prognostic features, such as pathologic stage and grade, have limited ability to predict the outcomes in Urothelial carcinoma of the bladder (UCB). The Immunohistochemical analysis of biomarker expression has provided insight into the molecular pathogenesis of UCB and offers the potential for improving clinical decision making. Numerous candidate immunohistochemical biomarkers for patients 
with UCB have been identified, with those relating to the cell cycle and apoptosis/cell proliferation being the most extensively studied [66].

a1-3/4 fucosylation at Asn 241 of $\beta$-haptoglobin is a novel marker for colon cancer: a combinatorial approach for development of glycan biomarkers, Aberrant glycosylation has been observed in many types of cancer, but the mechanism of glycosylation change is still poorly understood. In order to elucidate relationships between glycosylation and colon cancer progression, we analyzed glycosylation status of $\beta$-haptoglobin ( $\beta$-Hp) obtained from 46 cancer patients, 14 inflammatory bowel disease (IBD) patients, and 38 normal subjects. Aleuria aurantia lectin reactivity with cancer $\beta$-Hp was much higher than in the other two study groups. These results were confirmed by lectin blotting and microarray assay using other lectins directed to fucosyl residues. Levels of such glycans were correlated with stage of colon cancer progression [62].

Despite recent advances in treatment to cancer of different kinds, Rituximab, the first monoclonal antibody (MAb) evaluated in MM treatment, provided only very limited benefit, research is ongoing into a number of other MAbs directed against a variety of MM-related target antigens. Potential MAb targets in MM include growth factors and their receptors, other signalling molecules, and antigens expressed exclusively or predominantly on MM cells. MAb therapy involves a range of mechanisms, including antibody-dependent cellular cytotoxicity, complement-dependent cytotoxicity, interference with receptor-ligand interactions, and MAb conjugation to radioisotopes or toxins [64].

In many of the cases the Genes, gene instability or Microsatellite Instability were the main reasons where carcinogenesis has been escalated (as they are Biomarkers). For example, Genetic instability underlies the etiology of multistep gastric carcinogenesis. The $\mathrm{P}^{53}$ mutations observed in tumors represent the expression of such instability by allowing the accumulation of genetic alterations caused by multiple mechanisms. $\mathrm{P}^{53}$ gene mutation incites the pathogenesis of human gastric adenocarcinomas [5]; secondly, genes Encoding Enzymatic Activities Implicated in the Eicosanoid Cascade of Arachidonic Acid and their Receptors are Expressed at mRNA Levels in Human Meningiomas. By using real time PCR, their presence in human meningiomas. Genes encoding enzymatic activities implicated in the eicosanoid cascade are expressed in meningiomas. LOX- and COX-derived arachidonic acid metabolites might act on tumor growth not only by acting on cell growth but also by altering the local cytokine and/ or angiogenic networks [3]. Thirdly, Microsatellite Instability, to evaluate Microsatellite (MSI) instability at markers adjacent to Chromosomal loci involved in primary gastric lymphoma in relation to helicobacter eradication therapy. The incidence of complete remission post helicobacter pylori eradication was higher in Low grade MALT in comparison to MALTDLBCL and de novo DLBCL. The incidence of MSI is decrease post helicobacter pylori eradication in all subtypes. Remission of gastric lymphoma post Helicobacter eradication may associate with correction of MSI level [15]. Malignant Fibrous Histiocytoma is an Unusual Transformation from Benign to Malignant. Malignant Fibrous Histiocytoma (MFH) is one of the most common soft tissue sarcoma in adults and typically arises in the soft tissues of the extremities and retroperitoneum. Is one of the cancers resulted from the accumulation of metabolites resulting from genes Encoding Enzymatic Activities? It is a question still to approve [1].

\section{Biomarkers' for the HIV-resistant strains}

1. The Occurrence of HIV-1 Resistance Biomarker among Two Cohorts, approximately $1 \%$ of the Caucasians shows resistance to HIV1 infection conditioned by $32 \mathrm{bp}$ deletion in CCR 5 gene. Homozygotes are almost totally resistant, but heterozygote will have delayed progression to AIDS. Molecular analysis of CCR5del32 (c.794-925del) variant was carried out with PCR technique using primers Results of PCR amplification were visualized Analysis of the frequency of del32 variant in the CCR5 gene was assessed using the Pearson $\chi^{2}$ test. Occurrence of CCR5del32 biomarker does not reflect the incidence of HIV in the examined regions [9].

2. MTA1 Aids the AKT Pathway by Inhibiting Expression of a Key Regulator, PTEN Metastasis is the final result of actions of various genes, one of which is the Metastasis Associated 1 (MTA1) gene. MTA1 acts as part of a nucleosome remodeling and histone deacetylation complex and has been shown to aid metastasis by regulating many other molecules. there was a study that is there any possible relationship between MTA1 and the phosphatase and tensin analogue mutated on chromosome 10 (PTEN). PTEN is a tumour suppressor gene known to be mutated in several cancers. That resulted a finding that there a knockdown of MTA1 using siRNA, PTEN protein levels increased albeit its mRNA levels were unchanged. Further findings suggest that MTA1 and PTEN co-localize and co-Immunoprecipitate with each other [10].

\section{Growth factors' as the biomarkers' and their mechanism by Gene knock Down}

Epithelial growth factor (EGF) induced epithelio-mesenchymal transition (EMT) in epithelial ovarian cancer cells and concomitantly suppressed NGAL expression. A hypothesis is made that inhibition of NGAL expression induces EMT in these cells. Accordingly a method has been provided which is similar to the above gene knocking; NGAL knockdown was achieved using siRNA in ovarian cancer cell line OVCA 429. NGAL siRNA treated cells [ $\mathrm{n}=3$ ] were assayed for EMT markers such as migration and invasion assays, and E-cadherin, $\mathrm{N}$-cadherin, Snail, $\beta$-catenin and vimentin expression by Western blot. This concluded that direct NGAL inhibition by siRNA does not induce a mesenchymal transition of ovarian cancer cells [11]. In contrast to this the Molecular targeted therapy in ovarian cancer is also in existence, the identification of biomarkers predictive of response to targeted therapy is essential to avoid poor responses to potentially useful drugs in unselected trial populations. With some promising, albeit early, phase III data on the angiogenesis inhibitor bevacizumab, exciting new opportunities lie ahead with the ultimate goal of personalizing therapies to individual tumour profiles [93].

Genomic biomarkers were also been studied in predictive medicine by a simple interim analysis [73].

A genome wide association suggests two new biomarkers on the Lung cancer susceptibility loci: A significant study revealed that the combined analyses identified six well-replicated SNPs with independent effects and significant lung cancer associations [78].

Mucin: A glycosylated high molecular mass protein potential as novel clinical biomarkers for the diagnosis of various malignant tumors. Supported molecular matrix electrophoresis (SMME) is a new type of membrane electrophoresis that can be used to characterize mucins. In SMME, mucins migrate in a molecular matrix supported by membrane materials. In this technique an Immuno staining method 
has been developed for the identification of SMME-separated mucins. The present method allows simple and rapid characterization of mucins in terms of both glycans and core proteins. The method will be a useful tool for the exploration of mucin alterations associated with various diseases such as cancer [83].

\section{Prognostic studies}

Prognosis based on the biomarker's pathogenesis/pathogenicity (or) prognostic factors: To estimate the Biomarker's ability one should study the characteristics' of the biomarker. Biomarkers' abilities will be reviewed only when it has a certain notification of the property that means concomitantly the pathogenesis (chemical), and also physical properties in other words this is termed as the properties of the biomarker that get's notified when it gets in to laboratory bench and kept under observations in different aspects. The case studies like:

1. A New Stochastic Model of Retinoblastoma Involving both Hereditary and Non-hereditary Cancer Cases [12]. 2. Oral Submucous Fibrosis: A Clinico-Histopathological Comparative Study in Population of Southern Rajasthan [13]. The above two case studies were taken as the references for the time being.

\section{The following are the biomarkers novel to their existence and have got importance according their pathogenesis and are novel to their pathogenesis}

Biomarkers' for the Breast cancer: The proteomic analysis focused on the nuclear structure of human breast cancer tissue, which has been shown to be a promising tool for cancer biomarker development. The nuclear matrix composition of human breast cancer, benign controls and healthy controls was analyzed by high-resolution two-dimensional gel electrophoresis and mass spectrometry [75].

Here is the Recursive partitioning analysis in the prognostic scores to predict survival in patients with brain metastases from primary breast cancer. Recent data suggests that primary tumour characteristics might provide further important information.

The impact of primary tumour size, histological grade, hormone receptor status, number of lymph node metastases and Nottingham prognostic index (NPI) was evaluated together by performance status by uni- and multivariate analyses. Emerging prognostic factors such as NPI or triple-negative status might improve the models currently used by clinicians [14]. The Pathological examination has been the gold standard for diagnosis in cancer and its role has also included the elucidation of etiology, pathogenesis, clinicopathological correlation, and prognostication. The advent of newer technologies and the realization that breast cancer is heterogeneous has shifted the focus to prognostication, with increased attention being paid to the identification of morphological features and immunohistochemical markers of prognostic relevance [89].

Differential methylation hybridization analysis [90] was conducted using a chromosomal DNA mixture of ten normal and cancer tissue sets. A CpG microarray harboring 237,220 CpG sites of the whole genome was interrogated and the resulting methylation level differences, as well as the RNA expression differences, between the normal and cancer sets for selected genes were verified in breast cell lines by methylationspecific PCR and real-time PCR analyses. As a result, we identified and verified novel genes that were hypermethylated in breast cancer, such as NRN1, CA5B and RPIA. Pathway analysis of the genes with altered methylation patterns identified the involvement of a differentiation- related network of genes whose activity may be heavily regulated by STAT1 in breast tumorigenesis. The epigenetic dysregulation of cellular processes relevant to STAT1-dependent cellular differentiation may be intimately involved in breast carcinogenesis. These findings lend credence to the possibility of using tumor-specific alterations in methylation patterns as biomarkers in estimating prognosis and assessing treatment options for breast cancer.

Genetic instability as prognostic factors: As we studied about the genetic instability in the previous paragraph, $\mathrm{P}^{53}$ - the Molecular Guardian Crashes in Gastric Adenocarcinomas belongs to the genetic instability family - $\mathrm{P}^{53}$ gene mutation incites the pathogenesis of human gastric adenocarcinomas [7].

The cell- based quantification of molecular biomarkers in the histopathological specimens was developed as the prognostic factors: Here this is developed with the aim of advancing current visual estimation and pixel-level quantification methods. Distribution, morphology and biomarker state of each cell in the field, and allow multiple biomarkers to be quantified over specified cell types, regardless of their abundance [68].

Expression of the surface molecules on myeloma cells as the prognostic factors: Some mammalian plasma membrane proteins act as potential biomarkers and drug targets [84]. Expression of surface molecules on multiple myeloma cells and their potential role in pathogenesis, prognosis, and treatment will be discussed. Multiple myeloma cells express a number of surface molecules capable of activating downstream anti-apoptotic signaling pathways which could favor their growth and survival. Such cell surface molecules have been considered to be prognostic factors for multiple myeloma (MM), may become possible therapeutic targets in the future [16].

Another type of the molecules expressed on the surface. Those are Proteolytically-cleaved Fragments of Cell-surface Proteins from Live Tumor Cells Stimulate Anti-tumor Immune Response In vitro. Treating surface of live human breast adenocarcinoma cells (MCF7) with trypsin yields a digest that contains $0.7 \%$ of total cell protein. Despite this difference, the trypsin digest stimulates in cytotoxicity assays anti-tumor response which kills $10-40 \%$ more cancer cells than those stimulated with cells themselves. Trypsin digest contained essential antigens to induce an immune-mediated anti-tumor effect, and therefore, is candidate for anti-tumor vaccine development [17].

Despite several advanced methods recently developed for the analysis of hydrophobic proteins and proteins with posttranslational modifications, integral membrane proteins are still under-represented in plasma membrane proteome. Recent advances in proteomic investigation of plasma membrane proteins, defining their roles as diagnostic and prognostic disease biomarkers and as target molecules in disease treatment, are presented [84].

Expressing genes as the prognostic factors: Triple-negative breast cancers account for $10-17 \%$ of all breast carcinomas and there is considerable need for reliable prognostic markers to assist clinicians in making diagnostic and therapeutic management decisions. Altered ANXA7 (a novel pro-apoptotic tumor suppressor gene located on chromosome 10q21) protein levels are associated with a tumorprone phenotype in knockout mouse model and prognostically challenging aggressive forms of prostate and breast cancer. They used a retrospective prognostic tumor tissue microarray (TMA) technology in order to evaluate the ANXA7 immunoreactivity as possible diagnostic and/or prognostic marker of triple-negative breast cancer 
by immunoperoxidase assay using ANXA7 monoclonal antibody and they reported that ANXA7 may be a new prognostic marker or a target for improving the treatment efficiency of patients with triplenegative breast cancers [18]. Meanwhile, Loss of ANXA7 Expression is Associated with Poor Patient Survival in Ovarian Cancer. Loss of ANXA7 expression is also significantly associated with poor over-all patient survival. That concludes ANXA7 as a new prognostic marker or a target for improving the treatment efficiency of patients with ovarian cancers [19]. Protein expression patterns associated with advanced stage ovarian cancer. A comparative proteomic study on biopsies from patients with ovarian cancer to identify potential diagnostic/ prognostic biomarkers in both healthy and tumor tissue, Protein expression/identification was evaluated by $2-\mathrm{DE}$ and MS analysis: six proteins showed differential expression in tumoral interstitial fluid and tumor tissue compared to normal interstitial fluid and healthy tissue: five were found to be downregulated and identified as galectin 3, glutathione S-transferase A-2, retinol binding protein 1, phosphatidylethanolamine-binding protein and annexin 5 , while the calgranulin, was significantly upregulated in all pathological samples, this study suggests that calgranulin expression may be used as a diagnostic and/or prognostic biomarker [76].

Prognostic Value of Cell Cycle Proteins: Oral squamous cell carcinoma (OSCC) is a common malignancy that is characterized by a high degree of local aggression and lymph node metastasis. cyclin A, cyclin D1, p21 and p27 expression can be a valuable marker of poor prognosis and tumor aggressiveness in OSCC. cyclin A, cyclin D1, p21 and p27 expression can be a valuable marker of poor prognosis and tumor aggressiveness in OSCC [29].

Patterns of Multi-Biomarkers' for the tumor identification and premonition: The basic, clinical and translational research efforts have been directed to the identification of standard biomarkers associated with the degree of malignancy. There is a realistic need for the development of the early-stage cancer biomarkers supported by reliable and robust experimental data that can be readily applicable in the clinical practice. . Because it is well-known that several pathways and processes are altered in tumor cells, the concept of "single marker" in cancer results incorrect. Therefore, we can aim to analyze and discuss the proposal that the molecular profile of different genes or proteins in different altered tumor pathways must be established to provide a better global clinical pattern for cancer detection and prognosis [88].

Novel Diagnostic Method for the identification of Carcinoma of Unknown Primary (CUP) site: Molecular Tumor Profiling in the Diagnosis of Patients with Carcinoma of Unknown Primary Site, Retrospective Evaluation of Gene Microarray Assay: Molecular tumor profiling has potential importance in identifying the tissue of origin in patients with cancer of unknown primary (CUP). The study regarding this is retrospectively performed with the Tissue of Origin test, Gene microarray assay, on biopsy specimens from patients with CUP. Assay results were correlated with clinical and pathologic features, and with previous results using the Veridex 10-gene CUP assay, a molecular RTPCR assay designed to detect 6 primary sites. The Tissue of Origin test provided predictions of the primary site in $96 \%$ of patients with CUP. Additional trials are necessary to confirm the value of these assays [21].

A Superficial Colon Tumor Model Involving Subcutaneous Colon Translocation and Orthotopic Transplantation of Green Fluorescent Protein-Expressing Human Colon Tumor: Human colon cancer HT-29 cells were transduced with a pLPCX expression retroviral vector containing GFP and neomycin resistance genes. An incision was made on the cecal serosa followed by the implantation of a 1-mm tumor tissue to the cecum. For comparison, a conventional orthotopic transplantation model was established in a separate group of mice simultaneously. When tumor sizes reached $5 \mathrm{~mm}$ in diameter, half the mice in each model received 5-FU treatment. Primary tumor and metastases were monitored by fluorescent imaging or caliber measurement. The fluorescent superficial orthotopic transplantation colon model allows easier real-time visualization and more sensitive monitoring of tumor growth as well as convenient repeated sampling. It is a valuable orthotopic implantation model for evaluating colon cancer [22].

$\begin{array}{ccccc}\text { The } & \text { Molecular Alterations } & \text { Regarding Apopto- } \\ \text { sis in Hepatocellular Carcinoma Cells at a Glance: }\end{array}$ hepatocellular carcinoma (HCC) has been a grievous scourge of humanity for a long time. The current related articles and provide a further understanding about such molecular changes relevant to trigger imbalances in the regulation of apoptosis in HCC [23].

Serological Approach and Serum Metabolomics as the Novel diagnostic methods for the Gastro Intestinal Cancer: Conventional tumor markers are unsuitable for detecting carcinoma at an early stage and lack clinical efficacy and utility. In this study, we attempted to investigate the differences in serum metabolite profiles of gastrointestinal cancers and healthy volunteers using a metabolomic approach and searched for sensitive and specific metabolomic biomarker candidates. Even Proteomics based analysis for potential serum biomarkers' in Gall bladder [85] and in Cutaneous Malignant Melanoma (CMM) [96] were in progress. In this Targeted proteomics analysis High-throughput technologies can now identify hundreds of candidate protein biomarkers for any disease with relative ease. However, because there are no assays for the majority of proteins and de novo immunoassay development is prohibitively expensive, few candidate biomarkers are tested in clinical studies [86]. Human serum samples were obtained esophageal, gastric, and colorectal cancer patients and healthy volunteers $(n=12)$. A model for evaluating metabolomic biomarker candidates was constructed using multiple classification analysis. This Multiple classification analysis revealed that the variations in the levels of malonic acid and 1-serine largely contributed to the separation of esophageal cancer; gastric cancer was characterized by changes in the levels of 3-hydroxypropionic acid and pyruvic acid; and l-alanine, glucuronoic lactone and l-glutamine contributed to the separation of colorectal cancer. That revealed that some metabolites are more sensitive for detecting gastrointestinal cancer than conventional biomarkers [65]. As the screening programs have resulted in the reduction in the cancer mortality, thus this recent progress in reducing cancer mortality can largely be attributed to the implementation of screening programs. However, screening programs have not realized their full potential for the reduction of CRC mortality, because of poor adherence rates among patients. Serum biomarker screening tests offer the potential for improved patient adherence to CRC screening programs [69].

Immunological Approach in the Diagnosis: Monoclonal Antibodies as the biomarkers in the differential diagnosis of the pancreatic cancer, this study involves an immunological approach to establish novel monoclonal antibodies (mAbs) specific for the pancreatic juice from PC patients, which would be potentially useful in the diagnosis of pancreatic cancer PC [94].

Renal cell Carcinomas with Serum cell-free DNA as the Biomarker: Currently no serum biomarkers for the Renal Cell 
Carcinoma (RCC) were present. To study them the Preoperative serum samples with sporadic, solid renal tumors were analyzed by using Quantitative real-time polymerase chain reaction was used to assess total cell-free DNA (ring finger protein 185 [RNF185]) and CpG island methylation of Ras association domain family member $1 \mathrm{~A}$ (RASSF1A) von Hippel-Lindau (VHL), prostaglandin-endoperoxidase synthase 2 (PTGS2), and P16 (cyclin-dependent kinase inhibitor 2A). Associations with RCC, pathologic variables, and disease-specific survival were evaluated. The results indicated that cell-free DNA represents a novel serum-based diagnostic and prognostic biomarker for RCC. Total serum cell-free DNA levels and CpG island methylation of RASSF1A and VHL may be useful diagnostic biomarkers for RCC [82]. Another biomarker from the RCC is human leukocyte antigen class I, it identification and characterization has been done [97].

\section{Treatment of Cancer based on various Biomarkers}

New Targeted Therapies against (Breast) Cancer have been studied. Crucial pathways regulating the cell cycle, proliferation and survival of (breast) cancer cells have been investigated and aberrant components of these pathways have been exploited as new drug targets [24].

In vivo/ In vitro Anti-cancer activities of some compounds were studied accordingly, In vivo studies basing on different compounds. The quinazoline derivative 4-(4'-hydroxyphenyl)-amino-6,7dimethoxyquinazoline (WHI-P131/JANEX-1; CAS 202475-60-3) is a dual-function inhibitor of Janus kinase 3 (JAK3) and Epidermal Growth Factor (EGF) receptor kinase. A PEGylated liposomal nanoparticle formulation of GMP-grade WHI-P131 exhibited potent in vivo activity against breast cancer cells. Thus results demonstrate that the nanoparticle enabled drug delivery of WHI-P131 showed therapeutic potential against breast cancer [25]. Similar studies were made in in Vivo Cancer Targeting of Water-Soluble Taxol by Folic Acid Immobilization, results showed 2-3 month longer animal survival than that of taxol. Suggesting the conjugation with Dex and FA could provide an improvement in the anticancer therapy of taxol [26].

In Vitro studies basing on different Aspects: Fabrication of Lomustine Loaded Chitosan Nanoparticles by Spray Drying and in Vitro Cytostatic Activity on Human Lung Cancer Cell Line L132 is more efficient than the corresponding drug alone, due to the increased surface area lomustine loaded TPP and HMP crosslinked chitosan nanoparticles showed better anticancer activity [27].

Amplified Hypoxia Induced Tumor-Cell Death In vitro, a novel therapeutic approach of targeting tumor cells by depriving oxygen from them through increased hypoxia which surpasses their minimum requirement for oxygen; the latter achieved by interfering at the interface of oxygen diffusion between the blood vessels and tumor cells [28].

Radiation therapies: Intensity Modulated Radiation Therapy (IMRT) is widely accepted as an appropriate method to treat tumors at many different anatomic locations including lung. Monte Carlo (MC) dose calculation was used to evaluate the reliability of plan evaluation parameters compared to a pencil beam (PB) dose calculation for IMRT of the lung. The respective studies were made in accordance with the procedure. There can be large case-to-case differences between the parameters for both the targets and OARs. Accurate calculations can remove those remaining systematic errors from treatment plans compared to other combinatorial experiment calculations [29].

Relation between Tumor Size and Range of Motion in IMRT
Treatment Planning for Thoracic Lesions has been given to evaluate the relation between tumor size/volume, tumor range of motion, and healthy lung volume in light of radiotherapy motion management paradigm. Four patient data sets were considered in this investigation. Each patient underwent time resolved (4D) CT data scan. Midventilation CT data sets, with nominal lung volumes ranging from $\sim 3000 \mathrm{~cm} 3$ to $\sim 6000 \mathrm{~cm} 3$, were considered for treatment planning. Spheres with pre-specified radii were auto-contoured in the left lower lobes as simulated planning target volumes (PTVs) for each patient. Motion in superior-inferior direction was superimposed on the simulated spherical PTVs, such that motion-inclusive ITVs were generated. Nine-field IMRT treatment plans were created for all lung volumes, different combinations of simulated PTV spherical size and ranges of motion. Three dose levels of $60 \mathrm{~Gy}, 70 \mathrm{~Gy}$, and $80 \mathrm{~Gy}$ were utilized. The doses were prescribed to $95 \%$ of the ITV. Simulated PTV sizes and ranges of motion were varied until prescriptions were met, given that organs at risk (OARs) were spared. The OAR constraints were: $40 \mathrm{~Gy}$ to $1 \%$ of the cord and $30 \%$ of the heart, as well as $20 \mathrm{~Gy}$ and 30 Gy to $30 \%$ and $20 \%$ of benign lung, respectively. These constraints, representative for 2 Gy per fraction fractionation schemes, are commonly used clinically. The treatment plans were deemed clinically acceptable when standard deviation of the dose across the ITV was less than $3 \%$ of the prescription dose in addition to fulfillment of the OAR constraints [30].

Imaging techniques like MRI [58] and Molecular Imaging [59]. Have reported their use in the evaluation methods for the tumors like hepatic tumors and metastasis in different tissues respectively.

Bevacizumab (BV) is an anti-vascular endothelial growth factor antibody. When administered with other chemotherapeutic drugs, BVcombined regimens prolong survival of colorectal cancer patients. A II trial in order to confirm the pharmacokinetic parameters from 3-Tesla dynamic contrast-enhanced magnetic resonance imaging (DCE-MRI) as surrogate biomarkers of BV+FOLFIRI regimen efficacy in colorectal cancer with liver metastases [63]. This is the combinatorial application of radiation therapy and the chemotherapy. This combination chemotherapy plus bevacizumab lead the prognosis of patients diagnosed with metastatic colorectal cancer improved markedly over the last 12 years, increasing from 5 months with best supportive care to almost 2 years [87].

Saracatinib [32] Impairs Head and Neck Squamous Cell Carcinoma Invasion by Disrupting Invadopodia Function: Elevated Src kinase activity is linked to the progression of solid tumors, including head and neck squamous cell carcinoma (HNSCC). Src regulates HNSCC proliferation and tumor invasion, with the Src-targeted small molecule inhibitor saracatinib displaying potent anti-invasive effects in preclinical studies. However, the pro-invasive cellular mechanism(s) perturbed by saracatinib are unclear. The anti-proliferative and anti-invasive effects of saracatinib on HNSCC cell lines were therefore investigated in preclinical cell and mouse model systems. Saracatinib treatment inhibited growth, cell cycle progression and transwell Matrigel invasion in HNSCC cell lines [32]. and Sunitinib [51] is an orally administered multi-targeted tyrosine kinase inhibitor. The main targets are vascular endothelial growth factor receptor (VEGFR)-1, VEGFR-2, VEGFR-3, platelet-derived growth factor receptor (PDGFR)- $\alpha$ and PDGFR- $\beta$.

Gefitinib (of EFGR) is a small molecular inhibitor is associated with The type 1 insulin-like growth factor receptor (IGF1R) has been implicated in various aspects of adenoma development and metastasis [54]. 
Elotuzumab, an anti-CS1 MAb, has recently achieved clinically meaningful responses when combined with lenalidomide or bortezomib in patients with relapsed and relapsed/refractory Multiple Myeloma [64].

Pancreatic ductal adenocarcinoma (PDAC) is a highly malignant tumor, for which development of new biomarkers and therapeutic targets has become critical. The main cause of the poor prognosis in PDAC patients is the high invasive and metastatic potential of the cancer. Here, we report a new signaling pathway that was found to mediate the enhanced tumor cell motility in pancreatic cancer. Semaphorin $4 \mathrm{D}$ (Sema4D) is a ligand known to be expressed on different cell types, and has been reported to be involved in the regulation of immune functions, enhances tumor cell motility through binding its receptor, plexinB1, in pancreatic cancer providing a new insight into possible prognostic biomarkers and therapeutic targets in PDAC patients [52].

Evaluation of Early Response to Neoadjuvant Chemotherapy in Breast Cancer Patients by 18F-FDG and 99mTc-HL91 Imaging: By monitor breast cancer mass with $18 \mathrm{~F}$-fluorodeoxyglucose (FDG) and 99mTc-HL91 positron emission tomography (PET) imaging, this study compares the efficacy of the two imaging methods in evaluation of the early response to neoadjuvant chemotherapy in stage II and III breast cancer patients. In conclusion, we suggest that 18F-FDG imaging, but not 99mTc-HL91 imaging with hybrid PET/CT provides a costeffective method, which could be well accepted for the low-income population in developing countries, to monitor early tumor response after three cycles of neoadjuvant chemotherapy in stage II and III breast cancer by using the reduction of N/T ratio as predictor [33].

Urinary Peptides for the Detection of Muscle-Invasive Bladder Cancer There is a need for better biomarkers to detect both bladder cancer and distinguish muscle-invasive (stage $\mathrm{T} 2+$ ) from non-invasive (stage $\mathrm{Ta} / \mathrm{T} 1$ ) disease. They assessed whether MALDI-TOF-MS of the urine peptidome can achieve this [55]. For the cells' global analysis which is termed to be the Transcriptome analysis, genetic tagging with a reporter provides a powerful tool for identification and isolation of a specific mature cell type, cell like stem cell/ progenitor cells [56].

Some of them were transcriptional factors which act as the Novel biomarkers'. ENI- a transcription factor has a novel importance as a biomarker in Adenoid cystic carcinoma [57].

There are many detection technologies like PSA (prostrate- specific antigen) detection technologies have become controversial [53]. with the ethical issues, the ethical use of mandatory research biopsies, Increasingly, clinical trials incorporate translational research questions aimed at identifying biomarkers of response or resistance to agents under investigation. Biomarker assays can require tissue samples to be collected through a research biopsy before therapy, during treatment or at the time of tumor progression.

Nanoparticles and their Involvement in Synthetic Biology: As Richard Feynman said, "What I cannot create, I do not understand", the more we understand the complex behavior of biology, the more chance we will discover new drugs and control diseases. We envision that nanomedicine-based synthetic biology might revolutionize current biomedical science and technology in the future [34].

Binding and Internalization of Iron Oxide Nanoparticles Targeted To Nuclear Oncoprotein: The use of nanocarriers as drug delivery systems for chemotherapeutic agents can improve the overall pharmacological properties of commonly used drugs in chemotherapy. Recent work involving the incorporation of targeting ligands, to improve colocalization between the drug and cancer cells is also addressed. Furthermore, while the use of these nanocarriers in particular is the primary focus here, this review also contains a discussion on other commonly used nanocarriers in cancer therapy to include various polymer-based and polymer-protein conjugates. Finally, the possibility of using combinatorial approaches involving multiple surface modifi cations made to both liposomes and micelles in order to further improve their drug delivery capabilities is also discussed [35].

A targeted nanoconjugate, this include Mesoporous silica Nanoparticles as Drug Delivery System [36], is being developed for non-invasive detection of gene expression in cells expressing the JC virus oncoprotein, T-antigen, which has been associated with Medulloblastoma and other cancers. JC virus T-antigen localizes predominantly to the nucleus via a classical monopartite nuclear localization signal (NLS). An antibody fragment which recognizes JC virus $\mathrm{T}$-antigen was attached to cross-linked dextran coated iron oxide nanoparticles [37], The rainbow nanobubble mechanism has been studied in water and in living cells in vitro. This mechanism provided maximal selectivity of the nanobubble generation in both models and therefore, can the therapeutic selectivity and optical contrast of gold nanoparticles in a heterogeneous physiological microenvironment at cell level [38]. That states to be the potential synthetic biomarker development respectively.

\section{Combinatorial approach}

Irinotecan and BCNU-Impregnated Wafers Used in Combination, for the Treatment of Patients with Recurrent Glioblastoma Multiforme, This retrospective study examines the toxicity of the combination of intravenous Irinotecan, and BCNU (1,3-bis(2-chloroethyl)-1nitrosurea) impregnated wafers following implantation at the time of tumor recurrence in patients with Glioblastoma Multiforme (GBM). The combination of systemic Irinotecan along with local intratumoral $\mathrm{BCNU}$ is well tolerated and may be more efficacious than either treatment alone. Future prospective is to study designed to confirm the effectiveness of this combined treatment strategy [39]. [40].

The Future of Melanoma Therapy is the Combination Approach

The Future of all Cancer Therapies is of Combination Approach in treatment [40].

A Retrospective Analytical studies and related work: To assess tumor control and survival of the patients with laryngeal cancers who received chemoradiation or radiotherapy alone as definitive treatment. Patients with laryngeal cancers who received organ-saving treatment were enrolled in this trial. It seems that radiotherapy or chemoradiation can be appropriate alternative to total laryngectomy in laryngeal cancers [41].

Association of Butyric Acid Produced by Periodontopathic Bacteria with Progression of Oral Cancer In this study, the effects of $\mathrm{BA} /$ sodium butyrate $(\mathrm{NaB})$ on podoplanin expression, cell migration and epithelial-mesenchymal transition in OSCC cell lines were examined. BA/ $\mathrm{NaB}$ increases podoplanin expression and cell migration in certain OSCC cell lines, suggesting that the progression of periodontal disease may promote the progression of OSCC via a podoplanin-dependent pathway [42]. 


\section{Radio embolization technique}

Selective internal radiation therapy (SIRT), otherwise known as radio embolization is now becoming a common procedure performed for those patients with primary hepatic neoplasia such as hepatocellular carcinoma, Hepatocellular carcinoma (HCC), a hypervascular tumor, is one of the most common and lethal cancers worldwide. We previously showed that resveratrol, a dietary polyphenol, inhibits rat liver carcinogenesis through antioxidative and anti inflammatory mechanisms [43] and liver dominant metastatic disease such as in near endocrine disease, and colorectal carcinoma [44]. Metastatic colorectal cancer (mCRC) remains largely an incurable condition. Although progress has been made in expanding the number of available systemic agents, treatment after initial therapy has limited benefit. Radioembolization (RE) with radio labeled microspheres is an emerging treatment modality for mCRC [31]. The challenge in clinical decision making includes patient selection, timing and procedure, In the U.S. For patients undergoing intrahepatic therapies, procedure selection is based not only on disease bulk but also on disease location [45].

Hepatic brachytherapy using either resin or glass $90 \mathrm{Y}$ microspheres is an established therapy for unresectable primary and metastatic tumors. Unlike conventional brachytherapy, microsphere brachytherapy has no software currently available for pretreatment evaluation and radiation planning. Software estimates of radiation dose preserving critical spatial information in the liver and tumors appeared reasonable based on clinical outcomes. Further testing and refinement of the software interface is ongoing with plans to distribute it to research organizations [46]. Within the setting of hepatic neoplasia [primary and secondary], selective internal radiation therapy [SIRT], also known as Radioembolization has become an accepted procedure, incorporated into the armamentarium of multidisciplinary oncologic care. The procedure itself requires an understanding of mesenteric vascular anatomy, tumor vascular patterns, liver metabolism, and chemotherapy [47].

A number of technologies are in clinical development to improve local control of liver metastases and potentially improve overall survival. Radio-embolization (RE) is a technique for administering resin or glass microspheres that contain yttrium-90 to unresectable primary or secondary hepatic malignancies internally via the liver's arterial supply in a single procedure. Clinical trials of RE used with concomitant radio sensitizing chemotherapy have shown promising results in patients with metastatic colorectal cancer. In this article, the evidence base for combining RE with systemic chemotherapy in the first line therapy of metastatic colorectal cancer is appraised and the scientific rationale for combining RE with chemotherapy in first and subsequent lines of therapy is outlined. Clinical trials of RE and chemotherapy currently recruiting patients with metastatic colorectal cancer are discussed in detail and practical recommendations offered on how best to combine RE and systemic chemotherapy [48].

Although clinical outcomes have been encouraging with both technology platforms, second-generation radioembolic devices [utilizing either new processes of microsphere synthesis or different radioactive isotopes] are currently under development, or clinical study [59].

It is usually offered to patients with advanced liver cancers. However, surgical experience after Radioembolization is very limited to anecdotal cases mainly related to hepatocellular carcinoma.
Here we report our initial single center experience with Yttrium-90 microspheres Radioembolization as down staging and bridging method for hepatocellular carcinoma prior liver surgery, resection or liver transplantation [49].

\section{Intentional weight loss reduces cancer risk?}

Epidemiologic evidence points to obesity as a major risk factor for many cancers, including cancers of the breast, endometrium, colorectum, kidney, esophagus, and pancreas. Whether intentional weight loss might reduce this excess risk is not yet proven. Observational cohort studies for intentional weight loss, and for studies reporting changes in cancer-relevant risk factors of estrogens, SHBG, IGF-I, IGF binding proteins, and selected inflammatory markers (CRP, IL6 , and TNF- $\alpha$ ) and and randomized controlled trials of both dietary interventions and bariatric surgery all indicate fairly immediate reductions in cancer incidence following intentional weight loss [74].

\section{Analysis}

Analysis of the Novel Biomarkers' by optical biosensor systems: Many cancer types are characterized by poor survival and unpredictable therapy response. Easy-to-perform molecular analyses may help patient stratification and treatment tailoring. Several integrated devices have been proposed to overcome current analysis equipment limitations. They offer improved sensitivity and easy availability of parallel detection. Particularly, unlabelled optical biosensors combine the manifold advantages of integrated sensors (e.g. easy handling, portability and low-volume requirement) with detection of target molecules in their original form [98].

Analysis by Polychromatic Flow cytometry: Pediatric solid tumors depend upon angiogenesis for their growth and metastases. A new polychromatic flow cytometry (PFC) protocol has revealed circulating cells of hematopoietic and endothelial lineages from the peripheral blood (PB) of healthy individuals, and has defined the different cell types involved in the growth of tumor vasculature that are critical in angiogenesis. The description of successful identification of these hematopoietic and endothelial progenitor cells in both healthy children and children with solid tumors. In addition, this is a potential discovery of novel predictive biomarkers for future clinical trials [99].

\section{Conclusion}

This review on Biomarkers of cancer in the prognosis and diagnosis has got a conclusion that many types of cancers have been evolved in the recent times thence however the methods of detection have also been evolved; the detection methods of classical forms have been made a noted significance in the earlier days but, after the discovery of the biomarker like substances. The process of the detection methods had made simple yet somewhat complicated though. This review covers hypothetic methods for the implementation of one of the recent time biomarkers for the prognosis and obviously the diagnosis of the other cancers. This in turn provides a prophylactic and treatment techniques for the further study regarding various studies of various cancers in the body.

\section{Acknowledgements}

I sincerely thank Mrs. Mahalakshmi Ravikiran, for her support in writing a review article and all my friends in supporting me.

\section{References}

1. Bali A, Singh MP, Padmavathi, Khorate M, Ahmed J (2010) Malignant Fibrous Histiocytoma - An Unusual Transformation from Benign to Malignant. J Cancer Sci Ther 2: 053-057. 
Citation: Sai YRKM, Dattatreya A, Anand SY, Mahalakshmi D (2011) Biomarkers and their Role in Premonition, Interpretation and Treatment of Cancer. J Cancer Sci Ther S17. doi:10.4172/1948-5956.S17-002

2. Cruz M, Siden $\AA$, Tasat DR, Yakisich JS (2010) Are all Glioma Cells Cancer Stem Cells? J Cancer Sci Ther 2: 100-106

3. De Armas R, Durand K, Weinbreck N, Robert S Jean-Jacques Moreau, et al. (2010) Genes Encoding Enzymatic Activities Implicated in the Eicosanoid Cascade of Arachidonic Acid and their Receptors are Expressed at mRNA Levels in Human Meningiomas. J Cancer Sci Ther 1: 003-007.

4. Singh RK, Sudhakar A, Lokeshwar BL (2010) Role of Chemokines and Chemokine Receptors in Prostate Cancer Development and Progression. J Cancer Sci Ther 2: 089-094.

5. Abdullah S, Sameer S A, Dil-Afroze, Syeed N, Das BC, et al. (2010) P53 - The Molecular Guardian Crashes in Gastric Adenocarcinomas - A Study in an Ethnic Kashmiri Population. J Carcinogene Mutagene 1:106.

6. Bernstein C, Payne CM, Bernstein H (2011) Bile Acids: Promoters or Carcinogens in Colon Cancer? J Carcinogene Mutagene 2:101e.

7. Ezz Eldin AM, Alkareemy ER, Zedan A, Abdel Warith A, Zaky AH, et al. (2010) The Role of Anti-Helicobacter Pylori Therapy in Remission Induction of Primary Gastric Lymphoma by Analysis of Microsatellite Instability. J Carcinogene Mutagene 1:107.

8. Thomas S, Waterman P, Chen S, Marinelli B, Seaman M, et al. (2011) Development of Secreted Protein and Acidic and Rich in Cysteine (SPARC) Targeted Nanoparticles for the Prognostic Molecular Imaging of Metastatic Prostate Cancer. J Nanomedic Nanotechnol 2:112.

9. Siekierzynska A, Myszka A, Slezak R, Laczmanska I, Wnuk M (2011) The Occurrence of HIV-1 Resistance Biomarker Among Two Cohorts from Poland. J Mol Biomark Diagn 2:108.

10. Shimul S, Rajiv G (2010) MTA1 Aids the AKT Pathway by Inhibiting Expression of a Key Regulator, PTEN. J Cancer Sci Ther 2: 114-119.

11. Lim R, Lappas M, Ahmed N, Borregaard N, Quinn MA, et al. (2011) Effect of Silencing Neutrophil Gelatinase-Associated Lipocalin in Ovarian Cancer Cells on Epithelio-Mesenchymal Transition. J Mol Biomark Diagn 2:114.

12. Tan WY, Zhou H (2011) A New Stochastic Model of Retinoblastoma Involving both Hereditary and Non-hereditary Cancer Cases. J Carcinogene Mutagene 2:117.

13. Goel S, Ahmed J, Singh MP, Nahar P (2010) Oral Submucous Fibrosis: A Clinico-Histopathological Comparative Study in Population of Southern Rajasthan. J Carcinogene Mutagene 1:108.

14. Nieder C, Spanne O, Bilberg I, Dalhaug A (2010) Primary Tumour Characteristics as Potential Prognostic Factors in Brain Metastases from Breast Cancer. J Cancer Sci Ther 2: 070-073.

15. Abdullah S, Sameer S A, Dil-Afroze, Syeed N, Das BC, et al. (2010) P53- The Molecular Guardian Crashes in Gastric Adenocarcinomas - A Study in an Ethnic Kashmiri Population. J Carcinogene Mutagene 1:106.

16. Kotwica K, Cioch MB, Dmoszynska A (2011) Expression of Surface Molecules on Multiple Myeloma Cells and their Potential Role in Pathogenesis, Prognosis, and Treatment. J Mol Biomark Diagn 2:103.

17. Balashova EE, Lokhov PG (2010) Proteolytically-cleaved Fragments of Cellsurface Proteins from Live Tumor Cells Stimulate Anti-tumor Immune Response In vitro. J Carcinogene Mutagene 1:103.

18. Srivastava M, Eidelman O, Bubendorf L, Pollard HB (2011) ANXA7 Expression has Prognostic Impact for Patient Survival In Triple Negative Negative Breast Cancers. J Mol Biomark Diagn 2:110.

19. Srivastava M, Eidelman O, Bubendorf L, Sauter G, Pollard HB (2011) Loss of ANXA7 Expression is Associated with Poor Patient Survival in Ovarian Cancer. J Mol Biomark Diagn 2:113.

20. Buim MEC, Fregnani JH, Lourenço SV, Nagano CP, Carvalho AL (2011) Prognostic Value of Cell Cycle Proteins in Squamous Cell Carcinomas of the Oral Cavity. J Mol Biomark Diagn 2:111.

21. Hainsworth JD, Pillai R, Henner WD, Halks-Miller M, Lane CM, et al. (2011) Molecular Tumor Profiling in the Diagnosis of Patients with Carcinoma of Unknown Primary Site: Retrospective Evaluation of Gene Microarray Assay. J Mol Biomark Diagn 2:106.

22. Jin H, Yang Z, Wang J, Zhang S, Sun Y, et al. (2010) A Superfi cial Colon Tumor Model Involving Subcutaneous Colon Translocation and Orthotopic Transplantation of Green Fluorescent Protein-Expressing Human Colon Tumor. J Carcinogene Mutagene 1:104.
23. Yuan Y, Zhang YS, Qu XJ (2011) The Molecular Alterations Regarding Apoptosis in Hepatocellular Carcinoma Cells at a Glance. J Carcinogene Mutagene 2:118

24. Nicolin V, Bareggi R, Narducci P (2010) New Targeted Therapies Against Breast Cancer. J Carcinogene Mutagene 1:110.

25. Dibirdik I, Yiv S, Qazi S, Uckun FM (2010) In vivo Anti-Cancer Activity of a Liposomal Nanoparticle Construct of Multifunctional Tyrosine Kinase Inhibito 4-(4'-Hydroxyphenyl)-Amino-6,7-Dimethoxyquinazoline. J Nanomedic Nanotechnolo 1: 101

26. Nakamura J, Nakajima N, Matsumura K, Hyon SH (2011) In Vivo Cancer Targeting of Water-Soluble Taxol by Folic Acid Immobilization. J Nanomedic Nanotechnol 2:106.

27. Mehrotra A, Nagarwal RC, Pandit JK (2010) Fabrication of Lomustine Loaded Chitosan Nanoparticles by Spray Drying and in Vitro Cytostatic Activity on Human Lung Cancer Cell Line L132. J Nanomedic Nanotechnolo 1: 103.

28. Nair A (2010) Amplified Hypoxia Induced Tumor-Cell Death In vitro. J Carcinogene Mutagene 1:109.

29. Aljarrah K, Pawlicki T, Niemierko A, Mell LK, Jiang SB (2010) A Clinical Study of MLC-Based IMRT Lung Dose Calculation Accuracy on Plan Evaluation Parameters. J Cancer Sci Ther 2: 074-081.

30. Mihaylov IB, Lerma FA, Moros EG (2010) Relation Between Tumor Size and Range of Motion in IMRT Treatment Planning for Thoracic Lesions. J Cancer Sci Ther 2: 095-099.

31. Anwunobi AP, Emeje MO (2011) Recent Application of Natural Polymers in Nanodrug Delievery. J Nanomedic Nanotechnol S4:002.

32. Ammer AG, Kelley LC, Hayes KE, Evans JV, Lopez-Skinner LA, et al. (2009) Saracatinib Impairs Head and Neck Squamous Cell Carcinoma Invasion by Disrupting Invadopodia Function. J Cancer Sci Ther 1: 052-061.

33. Song WZ, Wang Z, Li LW, Chen JH, Wang J (2010) Evaluation of Early Response to Neoadjuvant Chemotherapy in Breast Cancer Patients by 18F-FDG and 99mTc-HL91 Imaging. J Nucl Med Radiat Ther 1:102.

34. Yun et al. (2011) Nanomedicine-based Synthetic Biology. J Nanomedic Biotherapeu Discover 2011, 1:1

35. Khan DR (2010) The Use of Nanocarriers for Drug Delivery in Cancer Therapy J Cancer Sci Ther 2: 058-062.

36. Douroumis (2011) Mesoporous silica Nanoparticles as Drug Delivery System J Nanomedic Nanotechnol 2011, 2:3

37. Knight LC, Romano JE, Krynska B, Faro S, Mohamed FB, et al. (2010) Binding and Internalization of Iron Oxide Nanoparticles Targeted To Nuclear Oncoprotein. J Mol Biomark Diagn 1:102.

38. Lukianova-Hleb EY, Oginsky AO, Shenefelt DL, Drezek RA, Hafner JH, et al. (2011) Rainbow Plasmonic Nanobubbles: Synergistic Activation of Gold Nanoparticle Clusters. J Nanomedic Nanotechnol 2:104.

39. Dewan S, Stopa E, Alderson L, Sampath P (2010) Irinotecan and BCNUImpregnated Wafers Used in Combination, for the Treatment of Patients with Recurrent Glioblastoma Multiforme. J Carcinogene Mutagene 1:101.

40. Ascierto PA (2011) The Future of Melanoma Therapy is the Combination Approach. J Mol Biomark Diagn 2:102e.

41. Shaghayegh K, Mahdi A, Ali K (2010) Larynx Preserving Treatments in the Early and Advanced Laryngeal Cancers; A Retrospective Analysis. J Cancer Sci Ther 1: 008-010.

42. Miyazaki Y, Kikuchi K, González-Alva P, Inoue H, Noguchi Y, et al. (2010) Association of Butyric Acid Produced by Periodontopathic Bacteria with Progression of Oral Cancer. J Cancer Sci Ther 2: 026-032.

43. Bishayee A, Petit DM, Samtani K (2010) Angioprevention is Implicated in Resveratrol Chemoprevention of Experimental Hepatocarcinogenesis. J Carcinogene Mutagene 1:102.

44. Burrill J, Hafeli U, Liu DM (2011) Advances in Radioembolization - Embolics and Isotopes. J Nucl Med Radiat Ther 2:107.

45. Anthony L (2011) Neuroendocrine Hepatic Tumors: Summary of Patient Selection, Response and Toxicity of Radioembolization in 281 Patients. J Nucl Med Radiat Ther 2:104. 
Citation: Sai YRKM, Dattatreya A, Anand SY, Mahalakshmi D (2011) Biomarkers and their Role in Premonition, Interpretation and Treatment of Cancer. J Cancer Sci Ther S17. doi:10.4172/1948-5956.S17-002

46. Kennedy A, Dezarn W, Weiss A (2011) Patient Specific 3D Image-Based Radiation Dose Estimates for 90Y Microsphere Hepatic Radioembolization in Metastatic Tumors. J Nucl Med Radiat Ther 2:111.

47. Liu DM, Cade D, Klass D, Loh C, McWilliams JP, et al. (2011) Interventional Oncology - Avoiding Common Pitfalls to Reduce Toxicity in Hepatic Radioembolization. J Nucl Med Radiat Ther 2:106.

48. Hill EJ, Sharma RA (2011) Multi-modality Therapy of Hepatic Metastases from Colorectal Carcinoma: Optimal Combination of Systemic Chemotherapy with Radio-embolization. J Nucl Med Radiat Ther 2:108. doi:10.4172/2155 9619.1000108

49. Ettorre GM, Vennarecci G, Santoro R, Miglioresi L, Lepiane P, et al. (2011) Experiences in Hepatic Surgery and Transplantation after Radioembolization. J Nucl Med Radiat Ther 2:109.

50. Sinclair, D, Dagg, SH, Smith, SG, et al. (1986) The incidence and possible relevance of Bence-Sones protein in the sera of patients with multiple myeloma Br J Haematol 62: 689-694

51. Yuasa T, Takahashi S, Hatake K, Yonese J, Fukui I (2011) Biomarkers to Predict Response to Sunitinib Therapy and Prognosis in Metastatic Renal Cell Cancer. Cancer Sci.

52. Kato S, Kubota K, Shimamura T, Shinohara Y, Kobayashi N, et al. (2011) Semaphorin 4D, a lymphocyte semaphorin, enhances tumor cell motility through binding its receptor, plexinB1, in pancreatic cancer. Cancer Sci.

53. Faulkner A (2011) Resisting the screening imperative: patienthood, populations and politics in prostate cancer detection technologies for the UK.Sociol Health IIIn.

54. Shaw PH, Maughan TS, Clarke AR (2011) Dual inhibition of epidermal growth factor and insulin-like 1 growth factor receptors reduce intestinal adenoma burden in the $\mathrm{Apc}(\mathrm{min} /+)$ mouse. $\mathrm{Br} \mathrm{J}$ Cancer.

55. Bryan RT, Wei W, Shimwell NJ, Collins SI, Hussain SA, et al. (2011) Assessment of High-Throughput High-Resolution MALDI-TOF-MS of Urinary Peptides for the Detection of Muscle-Invasive Bladder Cancer. Proteomics Clin Appl.

56. Wang J, Zhang H, Young AG, Qiu R, Argalian S, et al. (2011) Transcriptome Analysis of Neural Progenitor Cells by a Genetic Dual Reporter Strategy. Stem Cells.

57. Bell D, Bell A, Roberts D, Weber RS, El-Naggar AK (2011) Developmental transcription factor EN1-a novel biomarker in human salivary gland adenoid cystic carcinoma. Cancer

58. Lee CH, Braga L, de Campos RO, Semelka RC (2011) Hepatic tumor response evaluation by MRI. NMR Biomed.

59. McCann TE, Kosaka N, Turkbey B, Mitsunaga M, Choyke PL, et al. (2011) Molecular imaging of tumor invasion and metastases: the role of MRI. NMR Biomed.

60. Jamaspishvili T, Kral M, Khomeriki I, Vyhnankova V, Mgebrishvili G, et al (2011) Quadriplex model enhances urine-based detection of prostate cancer. Prostate Cancer Prostatic Dis.

61. Yang K, Chen XZ, Zhang B, Yang C, Chen HN, et al. (2011) Is CD133 a biomarker for cancer stem cells of colorectal cancer and brain tumors? A metaanalysis. Int J Biol Markers.

62. Park SY, Lee SH, Kawasaki N, Itoh S, Kang K, et al. (2011) a1-3/4 fucosylation at Asn 241 of $\beta$-haptoglobin is a novel marker for colon cancer: a combinatorial approach for development of glycan biomarkers. Int J Cancer.

63. Hirashima Y, Yamada Y, Tateishi U, Kato K, Miyake M, et al. (2011) Pharmacokinetic parameters from 3-Tesla DCE-MRI as surrogate biomarkers of antitumor effects of bevacizumab plus FOLFIRI in colorectal cancer with liver metastasis. Int J Cancer.

64. Richardson PG, Lonial S, Jakubowiak AJ, Harousseau JL, Anderson KC (2011) Monoclonal antibodies in the treatment of multiple myeloma. $\mathrm{Br} \mathrm{J}$ Haematol.

65. Ikeda A, Nishiumi S, Shinohara M, Yoshie T, Hatano N, et al. (2011) Serum metabolomics as a novel diagnostic approach for gastrointestinal cancer. Biomed Chromatogr.

66. Matsushita K, Cha EK, Matsumoto K, Baba S, Chromecki TF, Fajkovic H, et al. (2011) Immunohistochemical biomarkers for bladder cancer prognosis. Int $\mathrm{J}$ Urol.
67. Al-Kofahi Y, Lassoued W, Grama K, Nath SK, Zhu J, et al. (2011) Cellbased quantification of molecular biomarkers in histopathology specimens. Histopathology.

68. Creeden J, Junker F, Vogel-Ziebolz S, Rex D (2011) Serum tests for colorecta cancer screening. Mol Diagn Ther.

69. Vlaeminck-Guillem V, Bandel M, Cottancin M, Rodriguez-Lafrasse C, Bohbo JM, et al. (2011) Chronic prostatitis does not influence urinary PCA3 score. Prostate.

70. Nankivell P, Weller M, McConkey C, Paleri V, Mehanna H (2011) Biomarkers in laryngeal dysplasia: A systematic review. Head Neck.

71. Chen L, Fang B, Giorgianni F, Gingrich JR, Beranova-Giorgianni S (2011) Investigation of phosphoprotein signatures of archived prostate cancer tissue specimens via proteomic analysis. Electrophoresis.

72. Simon R (2011) Genomic biomarkers in predictive medicine. An interim analysis. EMBO Mol Med.

73. Byers T, Sedjo RL (2011) Does intentional weight loss reduce cancer risk? Diabetes Obes Metab.

74. Debald M, Franken S, Heukamp LC, Linke A, Wolfgarten M, et al. (2011) Identification of specific nuclear structural protein alterations in human breast cancer. J Cell Biochem.

75. Cortesi L, Rossi E, Casa LD, Barchetti A, Nicoli A, et al. (2011)Protein expression patterns associated with advanced stage ovarian cancer. Electrophoresis.

76. Garrido-Medina R, Díez-Masa JC, de Frutos M (2011) CE methods for analysis of isoforms of prostate-specific antigen compatible with online derivatization for LIF detection. Electrophoresis.

77. Hu Z, Wu C, Shi Y, Guo H, Zhao X, et al. (2011) A genome-wide association study identifies two new lung cancer susceptibility loci at 13q12.12 and 22q12.2 in Han Chinese. Nat Genet.

78. Koochekpour S (2011) DNA methylome and the complexity of discovering prostate cancer biomarkers. Asian J Androl.

79. Wong AM, Kong KL, Tsang JW, Kwong DL, Guan XY (2011) Profiling of Epstein-Barr virus-encoded microRNAs in nasopharyngeal carcinoma reveals potential biomarkers and oncomirs. Cancer.

80. Yeh MH, Tsai TC, Kuo HP, Chang NW, Lee MR, et al. (2011) Lentiviral shor hairpin RNA screen of human kinases and phosphatases to identify potentia biomarkers in oral squamous cancer cells. Int J Oncol.

81. de Martino M, Klatte T, Haitel A, Marberger M (2011) Serum cell-free DNA in renal cell carcinoma: A diagnostic and prognostic marker. Cancer.

82. Matsuno YK, Dong W, Yokoyama S, Yonezawa S, Saito T, et al. (2011) Improved method for immunostaining of mucin separated by supported molecular matrix electrophoresis by optimizing the matrix composition and fixation procedure. Electrophoresis. 32: 1829-1836

83. Rucevic M, Hixson D, Josic D (2011) Mammalian plasma membrane proteins as potential biomarkers and drug targets. Electrophoresis. 32: 1549-1564

84. Tan Y, Ma SY, Wang FQ, Meng HP, Mei C, et al. (2011) Proteomic-based analysis for identification of potential serum biomarkers in gallbladder cancer. Oncol Rep. 26: 853-859

85. Whiteaker JR, Lin C, Kennedy J, Hou L, Trute M et al. (2011) A targeted proteomics-based pipeline for verification of biomarkers in plasma. Nat Biotechnol. 29:625-34

86. Zinser-Sierra JW, Rodríguez-Ramírez S, Villalobos-Valencia R, RamírezMárquez M (2011) Use of bevacizumab in metastatic colorectal cancer: report from the mexican opinion and analysis forum on colorectal cancer treatment with bevacizumab (september 2009). Drugs R D. 11:101-111.

87. Rodríguez-Enríquez S, Pacheco-Velázquez SC, Gallardo-Pérez JC, MarínHernández A, Aguilar-Ponce JL (2011) Multi-biomarker pattern for tumor identification and prognosis. J Cell Biochem.

88. Leong AS, Zhuang Z (2011) The changing role of pathology in breast cance diagnosis and treatment. Pathobiology.78: 99-114.

89. Kim JH, Kang HS, Kim TW, Kim SJ (2011) Differential methylation hybridization profiling identifies involvement of STAT1-mediated pathways in breast cancer Int J Oncol. 39: 955-963. 
Citation: Sai YRKM, Dattatreya A, Anand SY, Mahalakshmi D (2011) Biomarkers and their Role in Premonition, Interpretation and Treatment of Cancer. J Cancer Sci Ther S17. doi:10.4172/1948-5956.S17-002

90. Ma Y, Zhang P, Yang J, Liu Z, Yang Z et al. (2011) Candidate MicroRNA biomarkers in human colorectal cancer: Systematic review profiling studies and experimental validation. Int $\mathrm{J}$ Cancer.

91. Izumiya M, Tsuchiya N, Okamoto K, Nakagama H (2011) Systematic exploration of cancer-associated microRNA through functional screening assays. Cancer Sci.

92. Kalachand R, Hennessy BT, Markman M (2011) Molecular targeted therapy in ovarian cancer: what is on the horizon? Drugs. 71: 947-967.

93. Hamabashiri M, Daichou A, Yasumoto M, Ogasawara S, Nishinakagawa T (2011) Novel monoclonal antibodies against pancreatic juice from pancreatic cancer patients and their possible application in differential diagnosis. Int $\mathrm{J}$ Mol Med. 28: 599-603.

94. Anderson KS, Wong J, D'Souza G, Riemer AB, Lorch J (2011) Serum antibodies to the HPV16 proteome as biomarkers for head and neck cancer. $\mathrm{Br}$ J Cancer. 104: 1896-1905.

95. Solassol J, Du-Thanh A, Maudelonde T, Guillot B (2011) Serum proteomic profiling reveals potential biomarkers for cutaneous malignant melanoma. Int J Biol Markers. 26: 82-87.
96. Seliger B, Dressler SP, Massa C, Recktenwald CV, Altenberend F et al. (2011) Identification and characterization of human leukocyte antigen class I ligands in renal cell carcinoma cells. Proteomics 11: 2528-2541.

97. Donzella V, Crea F (2011) Optical biosensors to analyze novel biomarkers in oncology. J Biophotonics. 4: 442-452.

98. Pradhan KR, Mund JA, Johnson C, Vik TA, Ingram DA (2011) Polychromatic flow cytometry identifies novel subsets of circulating cells with angiogenic potential in pediatric solid tumors. Cytometry B Clin Cytom.

99. Malu P, Anderson NL (2006) A List of Candidate Cancer Biomarkers for Targeted Proteomics. Biomarkers Insights 2: 1-48.

100. Chan DW, Sell S (1999) In Tietz Textbook of Clinical Chemistry (Eds, Burtis CA and Ashwood ER) W B Saunders Company, Philadelphia. pp: 722-749.

101.Bence-Jones, H 1847. Papers on Chemical Pathology: Lecture III. Lancet ii: 269-272.

102. Kyle, RA 1994: Multiple myeloma: How did it begin? Mayo Clin Proc. 